\title{
Variabilidad circadiana de la tasa de captura y la estructura de tallas en camarones e ictiofauna acompañante en la pesquería de arrastre del Mar Caribe de Colombia*
}

\author{
Luis O. Duarte ${ }^{1,2}$, Paul Gómez-Canchong ${ }^{1,2}$, Luis M. Manjarrés ${ }^{1}$, Camilo B. García ${ }^{3}$, Fabián D. \\ Escobar $^{1}$, Jairo Altamar ${ }^{1}$, Jorge E. Viaña ${ }^{4}$, Kenys Tejada ${ }^{1}$, Javier Sánchez $^{1} \&$ Félix Cuello $^{1}$ \\ ${ }^{1}$ Laboratorio de Investigaciones Pesqueras Tropicales, Universidad del Magdalena \\ Cra. 32 \# 22-08, Santa Marta, Colombia \\ ${ }^{2}$ Departamento de Oceanografía, Universidad de Concepción, Casilla 160-C, Concepción, Chile \\ ${ }^{3}$ Departamento de Biología, Universidad Nacional de Colombia, CECIMAR/INVEMAR \\ AA 1016 Santa Marta, Colombia \\ ${ }^{4}$ Instituto Colombiano de Desarrollo Rural, Edificio Concasa Piso 13, Cartagena, Colombia
}

\begin{abstract}
RESUMEN. A partir de muestreos realizados a bordo entre julio y septiembre de 2004, se examinaron los cambios asociados con la hora del día en las tasas de captura y estructura de tamaños de los camarones (Farfantepenaeus notialis, F. brasiliensis), las capturas incidentales, los descartes y las 12 especies de peces con mayores capturas en cada una de las dos zonas de pesca (norte y sur) en que opera la flota de arrastre de camarón en el Mar Caribe de Colombia. La tasa de captura de camarón fue significativamente mayor durante la noche, pero los individuos capturados en el día exhibieron mayor talla, lo cual ratifica la actividad nocturna que caracteriza a los camarones (Peneaidae). Especies de peces depredadores exhibieron mayores tasas de captura y menor representación de individuos grandes en horario diurno. La tasa de captura total (compuesta principalmente por especies descartadas de talla pequeña) resultó significativamente mayor en horas de la noche. La variabilidad circadiana observada en las tasas de captura y en la composición de tallas sugiere ritmos diurno - nocturno en la actividad de las especies examinadas y revela que los índices de abundancia pueden estar sesgados si los muestreos no son aleatorizados respecto a la hora del día. La elevada cantidad de individuos de talla pequeña descartados, evidencia una alta presión pesquera en la región y urge el establecimiento de medidas de manejo para mitigar el impacto de la pesca de arrastre.
\end{abstract}

Palabras clave: variabilidad circadiana, tasas de captura, estructura de tallas, camarón, fauna acompañante, Colombia.

\section{Circadian variability of catch rate and size structure for shrimp and fish bycatch from Colombian Caribbean trawl fishery*}

\begin{abstract}
Diel-associated changes of catch rates and size-structure of shrimp (Farfantepenaeus notialis and F. brasiliensis), incidental catches, discards and the twelve most caught fish species of each of the two shrimp trawling fishing zones (northern and southern) of the Colombian Caribbean Sea were examined. Data were obtained by on board observers between July and September 2004. Although individuals shrimps in daytime catches were larger in size, shrimp catch rates were significantly higher at nightime, wich confirm the nocturnal activity of the shimps (Penaeidae).Higher catch rates and few large individuals of predator fish species were observed during daytime. The total catch (mostly discarded, small-sized species) was significantly higher at night. Observed circadian variability in catch rates and size composition suggests diel rhythms in activity of examined species and indicates that the abundance indexes can be biased if sampling is not randomised by time of day. High amounts of discarded small sized individuals reveal a heavy fishing pressure in the region and indicate the urgent need for management measures to mitigate impacts of trawling.
\end{abstract}

Key words: circadian variability, catch rates, size structure, shrimp, bycatch, Colombia.

Autor corresponsal: Luis O. Duarte (lduarte@udec.cl)

* Trabajo presentado en el XXV Congreso de Ciencias del Mar de Chile y XI Congreso Latinoamericano de Ciencias del Mar (COLACMAR), realizados en Viña del Mar, entre el 16 y 20 de mayo de 2005. 


\section{INTRODUCCIÓN}

En el Mar Caribe de Colombia, la pesquería de arrastre de fondo ejerce presión sobre la comunidad demersal por la extracción del recurso objetivo (camarón) y se han registrado históricamente elevados niveles de captura de fauna acompañante (Duarte et al., 2004). Esta última es parte de la pesca ilegal, no registrada ni regulada y que se ha convertido en un tema central de la investigación pesquera internacional (Pitcher et al., 2002). Esta se divide en dos componentes: captura incidental y descarte. La captura incidental corresponde a individuos que, aunque no son objetivo de la pesquería, son utilizados y el descarte es la captura regresada al mar por razones económicas, legales o culturales (Alverson et al., 1994). Mundialmente, la pesca con redes de arrastre de camarón es la que más descartes produce, con efectos perjudiciales ampliamente reconocidos en la estructura y funcionamiento de los ecosistemas (Alverson et al., 1994; Hall, 1999). Un estudio retrospectivo de la estructura comunitaria y abundancia del camarón y de la fauna acompañante diagnosticó tendencias de disminución general en la biomasa de los recursos demersales impactados por la pesquería de arrastre en el norte del Mar Caribe de Colombia (Duarte et al., 2004).

La pesca de arrastre de camarón afecta las comunidades demersales mediante la remoción selectiva de individuos de un determinado rango de tallas, tanto de las especies objetivo como de las que sin ser objetivo son capturadas como fauna acompañante, y la modificación del hábitat (Hall, 1999). Como resultado se han observado cambios en la biomasa global, composición por especie y estructura de tallas (Pauly, 1988; Greenstreet \& Hall, 1996; Fogarty \& Murawsky, 1998). Las especies responden diferencialmente de acuerdo a sus características de historia de vida, interacciones tróficas, comportamiento y tipo de cambios generados en el hábitat físico (Bianchi et al., 2000). Los patrones recurrentemente observados en el comportamiento natural, incidentes en la disponibilidad de las especies a las redes de pesca y por tanto en sus respuestas al esfuerzo pesquero, corresponden a ritmos diarios de actividad, migración vertical y detección visual del arte (Sazima, 1986; Neilson \& Perry, 1990; Walsh, 1991; Walsh \& Hickey, 1993; Pillar \& Barange, 1997). En efecto, la confiabilidad de los índices de abundancia derivados de los arrastres de fondo y el tamaño de los individuos capturados dependen de una serie de factores, dentro de los que se cuenta la varia- bilidad circadiana en las tasas de captura (Michalsen et al., 1996; Benoit \& Swain, 2003; Yousif, 2003). La variabilidad en la eficiencia del arrastre debida al efecto de las embarcaciones o las artes puede ser controlada manteniendo esos factores constantes o ajustándolos mediante experimentos comparativos (Pelletier, 1998).

A medida que el nivel de explotación de un recurso o un área de pesca se incrementa, se tiende a reducir la selectividad del arte (moviendo la curva de selección hacia la izquierda), causando un incremento de la fauna acompañante y en la captura de individuos de tallas menores (Cook, 2003). Las medidas de mitigación usualmente incluyen modificaciones tecnológicas de las redes para aumentar su selectividad (Broadhurst, 2000), pero raramente se examina el impacto diferencial que la hora de captura puede tener sobre las especies y/o tallas disponibles para la pesquería.

La flota de arrastre que opera en el Mar Caribe de Colombia ha desarrollado preferencialmente faenas nocturnas; sin embargo, no se había evaluado el efecto de la hora en que se realiza la pesca sobre los rendimientos. El propósito de este estudio es examinar los cambios asociados con la hora del día en las tasas de captura y las estructuras de tallas de los camarones (Farfantepenaeus notialis, F. brasiliensis), de los descartes, de la captura incidental, de la captura total y de las especies de peces de la fauna acompañante que registraron mayores volúmenes de captura. Adicionalmente, se exploran diferencias en las distribuciones de talla entre las dos zonas de pesca del área de estudio. Se discuten las implicaciones de esta variabilidad circadiana en el rendimiento de la pesquería, el efecto selectivo sobre la fauna acompañante y el diseño de medidas de mitigación del impacto de la pesquería de arrastre.

\section{MATERIALES Y MÉTODOS}

Para efectos de manejo administrativo estatal, control operacional empresarial e investigaciones del camarón en el litoral Caribe de Colombia, se han considerado dos grandes zonas, norte y sur (Fig. 1), teniendo como límite el río Magdalena $\left(11^{\circ} 06^{\prime} \mathrm{N}\right.$, $74^{\circ} 50^{\prime} \mathrm{W}$ ). Las características de cada zona son distintas desde el punto de vista ecológico y climatológico, por lo cual es probable que los stocks objeto de explotación sean diferentes. En la zona norte se generan procesos de afloramiento costero tipo Ekman, con precipitaciones anuales bajas, en 

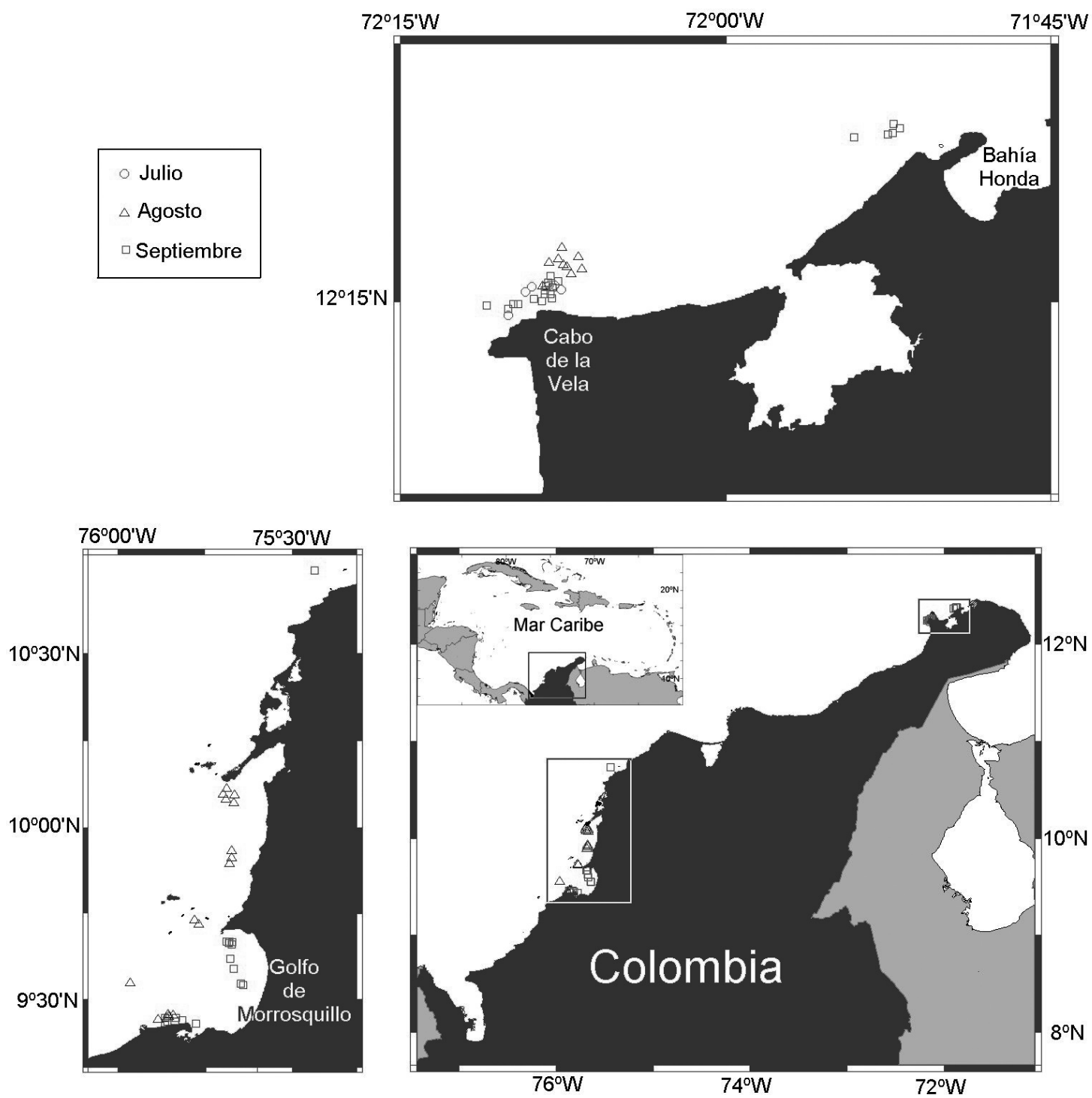

Figura 1. Ubicación de los lances muestreados por observadores a bordo de la flota de arrastre de camarón entre julio y septiembre de 2004, en las dos zonas de pesca del Mar Caribe de Colombia.

Figure 1. Location of hauls of the shrimp bottom trawl fleet sampled on board by observers between July and September 2004, in the two fishing zones of the Colombian Caribbean Sea.

tanto que la zona sur recibe mayor influencia de precipitaciones y descargas continentales que le dan un carácter estuarino (Andrade-Amaya, 2000; Manjarrés et al., 2003). Teniendo en consideración dicha zonación, mensualmente (entre julio y septiembre de 2004) se realizaron campañas de muestreo a bordo de la flota de arrastre de camarón, muestreándose un total de 67 lances de pesca ( 32 en la zona sur y 35 en la zona norte). La distribución espacial agregada de los lances correspondió a las zonas de pesca en que operó la flota, bien por la existencia de fondos apropiados para el arrastre o por preferencia de los capitanes de pesca (Fig. 1). En cada lance se analizó la composición, abundancia y tallas en la captura mediante el protocolo propuesto por Pauly (1983). Las medidas empleadas fueron longitud total para camarones y peces, y longitud del disco para rayas, usándose una precisión de $\pm 0,1 \mathrm{~cm}$. Para efectos de realizar los histogramas de frecuencia de tallas, la medida se aproximó al centímetro inferior. Debido 
a que la tripulación procesa los camarones a bordo no fue posible separar las capturas de cada una de las especies de camarón en la zona norte, por lo cual las tasas de captura presentadas son conjuntas. En la zona sur solo se registran capturas de F. notialis.

Con base en los resultados obtenidos, se seleccionaron doce taxa por zona de pesca, diez por su abundancia en peso y dos más por su abundancia en número (Diplectrum spp. y Selene setapinnis). Para probar la hipótesis nula que las tasas de captura $\left(\mathrm{kg} \cdot \mathrm{h}^{-1}\right)$ de camarones, descartes, captura incidental, captura total y cada uno de los taxa seleccionados por zona de pesca no difieren entre horas del día, se emplearon análisis de varianza no paramétricos de una vía Kruskal-Wallis (Kruskal \& Wallis, 1952). En el caso de encontrar diferencias estadísticas, se empleó la prueba de comparación múltiple Dunn-Sidak (Dunn, 1958; Sidak, 1967). Los lances se agruparon en cuatro rangos horarios, 04-10, 10-16, 16-22 y 22$04 \mathrm{~h}$, que representan las horas de amanecer, día, anochecer y noche, respectivamente.

El número de individuos capturado por hora de arrastre en cada rango de talla fue estimado mediante el método del estimador de proporción de la forma,

$$
\hat{R}=\sum_{i=1}^{n} a_{i} / \sum_{i=1}^{n} o_{i}
$$

donde $n$ son los lances muestreados, $a_{i}$ es el número de individuos capturados en el rango de talla en el lance i-ésimo y $o_{i}$ la duración del lance i-ésimo. $\mathrm{Si}$ bien este algoritmo ha sido empleado para la estimación de tasas de captura, resulta útil para obtener estimaciones de abundancia numérica usando el número de individuos capturado en lugar de peso capturado. Este estimador provee estimaciones insesgadas y confiables, particularmente cuando tanto la fauna acompañante como el camarón exhiben una alta variabilidad (Ye, 2002).

Las distribuciones de tallas de camarones, descartes, captura incidental, captura total y cada una de los taxa seleccionados fueron comparados entre zonas de pesca y entre los rangos horarios de cada zona de pesca mediante la prueba de independencia G (Sokal \& Rohlf, 2000), la cual corresponde al doble de la proporción de verosimilitud, que se aproxima a una distribución $\chi^{2}$ con $(\mathrm{r}-1) *(\mathrm{c}-1)$ grados de libertad y se calcula como sigue:

$$
\begin{gathered}
G=2 *\left[\sum_{i} \sum_{j} f_{i j} * \ln \left(f_{i j}\right)-\sum_{i} R_{i} * \ln \left(R_{i}\right)-\right. \\
\left.\sum_{j} C_{j} * \ln \left(C_{j}\right)+n * \ln (n)\right]
\end{gathered}
$$

donde $f_{i j}$ representa la frecuencia observada para el rango de talla i en la zona $\mathrm{j}, R_{i}$ representa la frecuencia total para el rango de talla $\mathrm{i}, C_{j}$ la frecuencia total para la zona $\mathrm{j}$, es decir, el tamaño de la muestra de esa zona y n el tamaño de la muestra.

\section{RESULTADOS}

Durante el periodo de estudio, los lances examinados correspondieron a una captura total (fauna y flora) de $8,44 \mathrm{t}$ en la zona norte y $5,96 \mathrm{t}$ en la zona sur. La duración de los lances fluctuó entre 3,2 y 8 h (5,6 $\mathrm{h}$ en promedio) en la zona norte y entre 1,9 y 5,8 $\mathrm{h}$ ( $4 \mathrm{~h}$ en promedio) en la zona sur. Once de los 35 lances en la zona norte fueron realizados en horario completamente diurno, mientras que en la zona sur todos los lances se efectuaron en horas de la noche, amanecer o atardecer. Este esquema de muestreo responde al régimen operacional de la flota.

Se capturó un elevado número de especies. En la zona norte se registraron 223 grupos taxonómicos de fauna acompañante y en la sur 180. Algunos taxa dominantes, en términos de tasa de captura, resultaron comunes para las dos zonas, destacándose Lutjanus synagris (pargo rayado), Synodus spp. (peces lagarto) y Syacium spp. (lenguados). Otros taxa solo fueron dominantes en una zona, como Cathorops spixii (bagre) y Lepophidium spp. (perlas) en la zona sur, y Micropogonias furnieri (corvina) y Orthopristis ruber (coroncoro) en la zona norte (Tablas 1 y 2).

En la zona norte, las tasas de captura de camarón (Farfantepenaeus notialis y F. brasiliensis en conjunto) fueron significativamente mayores $(\mathrm{p}<0,05)$ durante la noche (22-04 h) que en el día (10-16 h). Por el contrario, las tasas de captura de varios depredadores como L. synagris y M. furnieri, así como la captura incidental, resultaron mayores durante el horario diurno (10-16 h) (Tabla 1). En la zona sur no se registraron diferencias en las tasas de captura de camarón (F.notialis) entre los horarios muestreados, pero la tasa de captura total y de descartes fue mayor al atardecer (16-22 h) que al amanecer (04-10 $\mathrm{h}$ ), en tanto que peces demersales pequeños, como Lepophidium spp., Porichthys plectrodon (pez sapo) y Prionotus punctatus (pez gallina), registraron tasas de captura significativamente mayores en horas de la noche (22-04 h) que en otros horarios (Tabla 2).

El total de registros de tallas fue 67.038 ejemplares de $F$. notialis (32.335 en la zona norte y 34.703 en la zona sur), 20.755 de F. brasiliensis (solo capturados en la zona norte) y 80.803 registros de talla de 
peces (34.580 en la zona norte y 46.223 en la zona sur). La distribución de frecuencia de tallas de $F$. notialis indica mayor representación de individuos pequeños $(12 \mathrm{~cm})$ en la zona sur y se registró una diferencia significativa en la estructura de tallas entre las dos zonas (Fig. 2). La mayor proporción de individuos de F. brasiliensis en las capturas de la zona norte se concentró entre 12 y $15 \mathrm{~cm}$ LT (Fig. 2 y Tabla 3).

Se encontraron diferencias estadísticas $(\mathrm{p}<0,05)$ en la estructura de tallas de las capturas totales (multiespecíficas) entre las zonas de pesca, con una mayor proporción de tallas pequeñas (descartes) en la zona sur y mayor proporción de tallas grandes (incidental) en la zona norte $(\mathrm{p}<0,01)$. La distribución de tallas resultó ser bimodal en la zona norte y unimodal en la zona sur (Fig. 3). La mayoría de especies examinadas son pequeñas (no comerciales) y habitualmente descartadas en ambas zonas de pesca, como es el caso de P. plectrodon, Syacium spp. y Synodus spp. (Fig. 4, Tabla 4). Las fracciones de tamaño capturadas de las especies comerciales, como L. synagris, M. furnieri y Diapterus spp. (mojarras), resultaron significativamente diferentes entre las zonas de pesca $(\mathrm{p}<0,01)$, predominando tallas pequeñas descartadas en la zona sur y tallas grandes comercializadas (incidental) en la zona norte. En general, las especies seleccionadas presentaron una tendencia de mayores tamaños en la zona norte, con excepción Lepophidium spp., Selene setapinnis (jorobado), Upeneus parvus (salmonete), que exhibieron un patrón inverso (Fig. 4, Tabla 4). Algunas especies fueron capturadas sólo en una de las dos zonas, viz. C. spixii en la zona sur y Archosargus rhomboidalis (sargo) y $O$. ruber en la zona norte.

Tabla 1. Tasas de captura promedio $\left(\mathrm{kg}^{\circ} \mathrm{h}^{-1}\right)$ de los grupos analizados en la zona de pesca norte del Mar Caribe de Colombia entre julio y septiembre de 2004. p, nivel de significancia en la prueba Kruskal-Wallis. Pares p $<0,05$, comparaciones con un nivel de significancia fue inferior al $5 \%$ en la prueba Dunn-Sidak. La categoría camarones incluye $F$. notialis y $F$. brasiliensis. Las especies de peces están ordenadas de acuerdo a su importancia en peso dentro las capturas.

Table 1. Average catch rates $\left(\mathrm{kg}^{-1} \mathrm{~h}^{-1}\right)$ of the analysed groups in the northern fishing zone of Colombian Caribbean Sea between July and September 2004. p, significance level of the Kruskal-Wallis test. Pares p $<0.05$, pair comparisons with significance level lower than 5\% in the Dunn-Sidak test. Camarones (shrimps) include $F$. notialis and $F$. brasiliensis. Fish species are sorted by their importance in weight over the total catches.

\begin{tabular}{|c|c|c|c|c|c|c|}
\hline & Amanecer & Día & Atardecer & Noche & $\mathbf{p}$ & $\begin{array}{c}\text { Pares } \\
\mathbf{p}<\mathbf{0 , 0 5}\end{array}$ \\
\hline Número de lances & 6 & 7 & 12 & 10 & & \\
\hline Captura total & 59,48 & 131,72 & 65,64 & 50,58 & 0,355 & \\
\hline Camarones & 6,82 & 2,21 & 6,99 & 6,69 & 0,050 & $2 \mathrm{vs} 4$ \\
\hline Descartes & 43,29 & 69,94 & 47,32 & 35,97 & 0,359 & \\
\hline Captura incidental & 10,31 & 61,2 & 9,93 & 6,34 & 0,048 & $2 \mathrm{vs} 4$ \\
\hline Lutjanus synagris & 1,35 & 23,89 & 1,17 & 0,92 & 0,050 & $2 \mathrm{vs} 4$ \\
\hline Diapterus spp. & 0,72 & 13,15 & 1,95 & 1,10 & 0,007 & $1 \mathrm{vs} 2 ; 2 \mathrm{vs} 4$ \\
\hline Micropogonias furnieri & 2,98 & 6,59 & 1,53 & 0,74 & 0,480 & $2 \mathrm{vs} 4$ \\
\hline Orthopristis ruber & 1,84 & 1,81 & 4,10 & 1,31 & 0,745 & \\
\hline Synodus spp. & 5,52 & 4,82 & 1,19 & 1,12 & 0,002 & 1vs3; 1vs4; 2vs3 \\
\hline Archosargus rhomboidalis & 1,19 & 8,51 & 0,16 & 0,04 & 0,001 & $2 \mathrm{vs} 3 ; 2 \mathrm{vs} 4$ \\
\hline Syacium spp. & 2,34 & 3,16 & 1,86 & 1,20 & 0,095 & \\
\hline Haemulon aurolineatum & 1,12 & 3,65 & 2,68 & 0,15 & 0,383 & \\
\hline Prionotus punctatus & 2,19 & 1,27 & 1,27 & 0,84 & 0,844 & \\
\hline Upeneus parvus & 0,56 & 0,60 & 1,66 & 0,70 & 0,819 & \\
\hline Diplectrum spp. & 0,66 & 0,21 & 0,40 & 1,25 & 0,235 & \\
\hline Selene setapinnis & 0,88 & 0,84 & 0,28 & 0,25 & 0,111 & \\
\hline
\end{tabular}


Tabla 2. Tasas de captura promedio $\left(\mathrm{kg}^{-h^{-1}}\right)$ de los grupos analizados en la zona de pesca sur del Mar Caribe de Colombia entre julio y septiembre de 2004. p, nivel de significancia en la prueba Kruskal-Wallis. Pares p $<0,05$ comparaciones en las cuales en nivel de significancia fue inferior al $5 \%$ en la prueba Dunn-Sidak. Las especies de peces están ordenadas de acuerdo a su importancia en peso dentro de las capturas. No se realizaron lances en horario completamente diurno.

Table 2. Average catch rates $\left(\mathrm{kg}^{-h^{-1}}\right)$ of the analysed groups in the southern zone of Colombian Caribbean Sea between July and September 2004. p, significance level of the Kruskal-Wallis test. Pares p < 0.05 pair comparisons with significance level lower than $5 \%$ in the Dunn-Sidak test. Fish species are sorted by their importance in weight over the total catches. There is no hauls during daytime.

\begin{tabular}{|c|c|c|c|c|c|}
\hline & Amanecer & Atardecer & Noche & $\mathbf{p}$ & $\begin{array}{c}\text { Pares } \\
\mathbf{p}<\mathbf{0 , 0 5}\end{array}$ \\
\hline Número de lances & 9 & 12 & 11 & & \\
\hline Captura total & 41,13 & 73,97 & 64,62 & 0,009 & $1 \mathrm{vs} 3$ \\
\hline Farfantepenaeus notialis & 5,51 & 7,57 & 6,76 & 0,386 & \\
\hline Descartes & 32,36 & 54,62 & 46,88 & 0,042 & $1 \mathrm{vs} 3$ \\
\hline Captura incidental & 3,90 & 10,44 & 9,57 & 0,124 & \\
\hline Cathorops spixii & 3,01 & 13,76 & 9,90 & 0,124 & \\
\hline Lepophidium spp. & 1,26 & 3,77 & 4,22 & 0,025 & $1 \mathrm{vs} 3 ; 1 \mathrm{vs} 4$ \\
\hline Synodus spp. & 3,71 & 1,92 & 1,73 & 0,104 & \\
\hline Dasyatis spp. & 0,53 & 2,07 & 2,40 & 0,316 & \\
\hline Cynoscion ocoupa & 0,86 & 0,33 & 2,41 & 0,872 & \\
\hline Porichthys plectrodon & 2,25 & 1,33 & 0,19 & 0,012 & $1 \mathrm{vs} 4$ \\
\hline Prionotus punctatus & 0,54 & 1,92 & 1,93 & 0,050 & $1 \mathrm{vs} 4$ \\
\hline Syacium spp. & 0,97 & 1,33 & 1,33 & 0,223 & \\
\hline Lutjanus synagris & 0,58 & 1,43 & 0,62 & 0,689 & \\
\hline Eucinostomus spp. & 1,12 & 1,44 & 1,49 & 0,613 & \\
\hline Diplectrum spp. & 0,98 & 0,44 & 0,20 & 0,003 & $1 \mathrm{vs} 4$ \\
\hline Selene setapinnis & 0,21 & 0,23 & 0,04 & 0,098 & \\
\hline
\end{tabular}

Tabla 3. Estadísticos descriptivos de las tallas de las especies de camarones y pesca acompañante registradas en las zonas de pesca del Mar Caribe de Colombia entre julio y septiembre de 2004.

Table 3. Descriptive statistics of sizes of shrimp species and bycatch recorded in the fishing zones of Colombian Caribbean Sea between July and September 2004.

\begin{tabular}{|c|c|c|c|c|c|c|c|}
\hline & \multicolumn{2}{|c|}{$\begin{array}{c}F . \\
\text { notialis }\end{array}$} & \multirow{2}{*}{$\begin{array}{c}F . \\
\text { brasiliensis } \\
\text { Norte }\end{array}$} & \multicolumn{2}{|c|}{ Pesca incidental } & \multicolumn{2}{|c|}{ Descarte } \\
\hline & Norte & Sur & & Norte & Sur & Norte & Sur \\
\hline Promedio $(\mathrm{cm})$ & 14,1 & 13,8 & 14,1 & 21,2 & 18,9 & 11,6 & 11,7 \\
\hline Moda $(\mathrm{cm})$ & 13,0 & 12,0 & 13,0 & 18,0 & 16,0 & 11,0 & 9,0 \\
\hline Máximo (cm) & 21,0 & 20,0 & 21,0 & 42,0 & 67,0 & 41,0 & 41,0 \\
\hline Mínimo (cm) & 8,0 & 7,0 & 8,0 & 14,0 & 7,0 & 1,0 & 3,0 \\
\hline Desviación estándar & 10,0 & 9,0 & 2,6 & 5,6 & 6,8 & 5,6 & 4,8 \\
\hline Rango (cm) & 13,0 & 13,0 & 13,0 & 28,0 & 60,0 & 40,0 & 38,0 \\
\hline
\end{tabular}




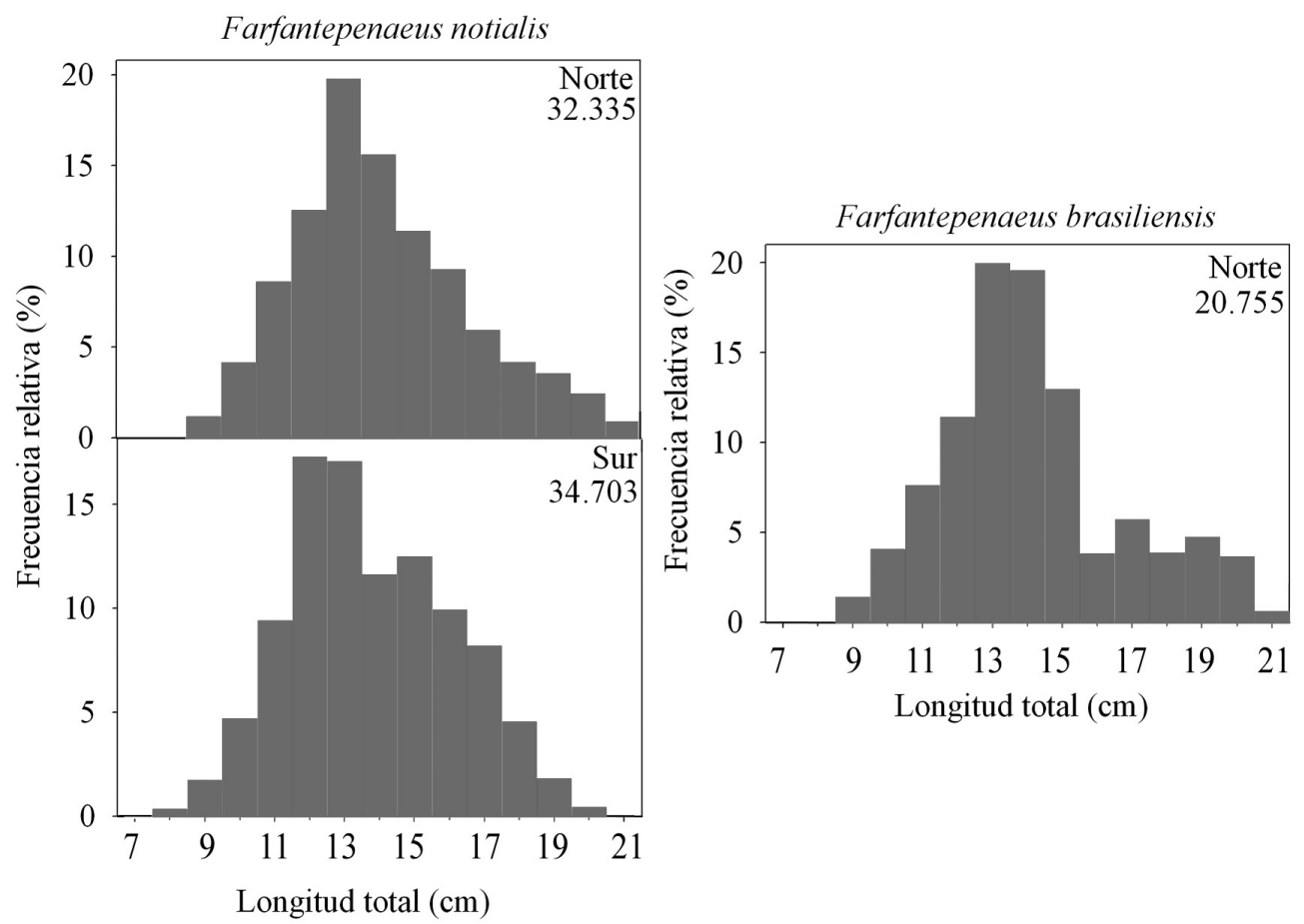

Figura 2. Estructuras de tallas de las especies de camarón (Farfantepeneus notialis, F. brasiliensis) registradas en las dos zonas de pesca. En $F$. notialis se detecto una diferencia significativa en la distribución de tallas entre las zonas mediante la prueba G. Los valores en cada pares indican el número de individuos observados en cada caso.

Figure 2. Size structures of shrimp species (Farfantepeneus notialis, F. brasiliensis) recorded in the two fishing zones. Significant difference of size distributions between zones was detected with $G$ test for $F$. notialis. Values in each chart indicate number of observed individuals.

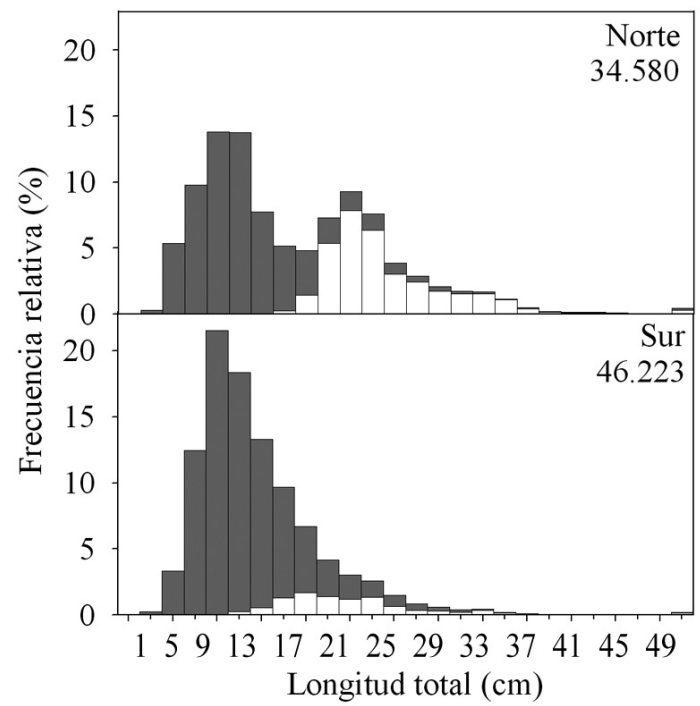

Figura 3. Estructuras de tallas de la comunidad de peces registradas en las dos zonas de pesca. Las barras oscuras representan la fracción de individuos descartada por intervalo de talla.

Figure 3. Size structures of fish community recorded in the two fishing zones. Shadow bars indicate percentage of discarded individuals by size interval. 
Tabla 4. Porcentaje de individuos descartados y orden de importancia de cada especie en términos del número de individuos capturados (N) y del peso (P) en cada zona de pesca. Comparaciones, resultados de las pruebas de independencia $\mathbf{G}$ para detectar diferencias entre las distribuciones de talla. ** Diferencia significativa de las distribuciones de tallas entre zonas para el total de los individuos capturados (G1), los individuos descartados (G2) y los individuos utilizados (incidentales) (G3). Comunidad, resultados del porcentaje de individuos descartados y de las comparaciones de la estructura de tallas de la comunidad de peces.

Table 4. Pertentage of individuals discarded and importance of each species in terms of the number of caught individuals $(\mathrm{N})$ and weight (P) for each fishing zone. Comparaciones, results of the $\mathrm{G}$ indepency test for to dectect differences between size structures. ** Significant difference of size distributions between zones for total individuals (G1), discarded individuals (G2), and used individuals (incidentals) (G3). Comunidad, results of discarded individuals and comparisons of the size structure of the fish community.

\begin{tabular}{|c|c|c|c|c|c|c|c|}
\hline & \multicolumn{3}{|c|}{ Norte } & \multicolumn{3}{|c|}{ Sur } & \multirow[b]{2}{*}{ Comparaciones } \\
\hline & $\begin{array}{c}\text { Descarte } \\
(\%)\end{array}$ & $\mathbf{N}$ & $\mathbf{P}$ & $\begin{array}{c}\text { Descarte } \\
(\%)\end{array}$ & $\mathbf{N}$ & $\mathbf{P}$ & \\
\hline Comunidad & 90,7 & & & 97,8 & & & $\mathrm{G} 1 * *, \mathrm{G} 2 * *, \mathrm{G} 3 * *$ \\
\hline Archosargus rhomboidalis & 2,9 & 18 & 7 & & & & NA \\
\hline Cathorops spixii & & & & 98,8 & 2 & 1 & NA \\
\hline Diapterus spp. & 17,9 & 9 & 2 & 96,5 & 9 & 12 & NA \\
\hline Diplectrum spp. & 100,0 & 4 & 15 & 100,0 & 8 & 10 & $\mathrm{G} 2 * *$ \\
\hline Eucinostomus spp. & 100,0 & 6 & 10 & 100,0 & 6 & 7 & $\mathrm{G} 2 * *$ \\
\hline Haemulon aurolineatum & 100,0 & 8 & 8 & & & & NA \\
\hline Lepophidium spp. & 100,0 & 14 & 36 & 96,7 & 3 & 3 & $\mathrm{G} 2 * *$ \\
\hline Lutjanus synagris & 5,0 & 10 & 1 & 70,5 & 16 & 17 & $\mathrm{G} 1 * *, \mathrm{G} 2 * *, \mathrm{G} 3 * *$ \\
\hline Micropogonias furnieri & 15,6 & 17 & 4 & 72,6 & 45 & 40 & NA \\
\hline Orthopristis ruber & 100,0 & 13 & 5 & & & & NA \\
\hline Porichthys plectrodon & 100,0 & 19 & 42 & 100,0 & 4 & 6 & $\mathrm{G} 2 * *$ \\
\hline Prionotus punctatus & 100,0 & 12 & 13 & 100,0 & 5 & 5 & $\mathrm{G} 2 * *$ \\
\hline Selene setapinnis & 99,8 & 5 & 18 & 72,6 & 45 & 40 & NA \\
\hline Syacium spp. & 100,0 & 1 & 9 & 100,0 & 1 & 4 & $\mathrm{G} 2 * *$ \\
\hline Synodus spp. & 100,0 & 2 & 6 & 100,0 & 7 & 2 & $\mathrm{G} 2 * *$ \\
\hline Upeneus parvus & 100,0 & 7 & 14 & 79,5 & 24 & 26 & $\mathrm{G} 1 * *, \mathrm{G} 2 * *$ \\
\hline
\end{tabular}

En la zona norte, la estructura de tamaños de $F$. brasiliensis y $F$. notialis difirió entre los horarios examinados (Tabla 5) con una menor representación de las tallas más pequeñas en horario diurno (10-16 h). En esta misma zona, diferencias estadísticas entre los rangos horarios examinados fueron detectadas en las distribuciones de frecuencias de tallas de la captura total, descarte y captura incidental (Tabla 5). Las capturas totales en la zona norte estuvieron homogéneamente distribuidas en un amplio rango de tallas (4 a $22 \mathrm{~cm}$ ) en horario diurno (10-16 h), en tanto que al amanecer y en la noche las tallas se concentraron en tallas pequeñas (4 a $12 \mathrm{~cm}$ ) (Fig. 5). En general, la distribución de frecuencias de tallas en los taxa de peces analizados en la zona norte fueron diferentes estadísticamente entre los rangos horarios examinados (Tabla 5, Fig. 6). Sólo se encontraron algunas excepciones. Las distribuciones de tallas de L. synagris y M. furnieri no fueron significativamente diferentes durante el atardecer y la noche. Una mayor representación de tallas pequeñas de estas especies se registró en horas diurnas y los individuos más grandes fueron capturados durante el amanecer, el atardecer y la noche. Las estructuras de tamaños de Diapterus spp. y A. rhomboidalis observadas en horas diurnas fueron significativamente diferentes a las de los demás rangos horarios por estar desplazadas hacia las tallas pequeñas (Tabla 5, Fig. 6). Los ejemplares 

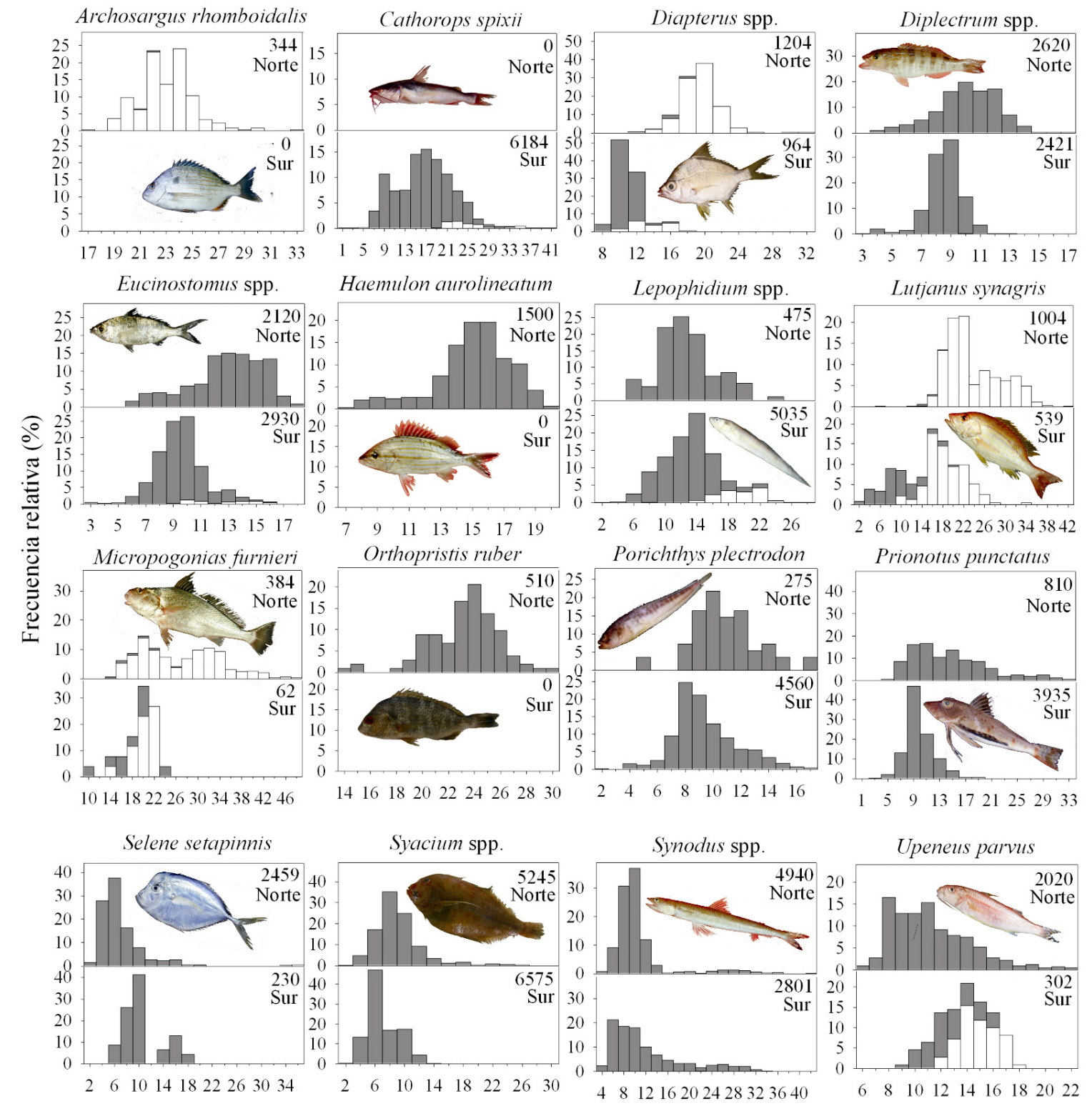

Figura 4. Estructura de tallas de los taxa de peces que dominaron las capturas en las dos zonas de pesca. Las barras oscuras representan la fracción de individuos descartada por intervalo de talla.

Figure 4. Size structures of dominant fish taxa of the community in the two fishing zones. Shadow bars indicate percentage of discarded individuals by size intervals.

de mayor talla en las especies pequeñas (Syacium spp., S. setapinnis, U. parvus, Diplectrum spp.) у medianas (Synodus spp., O. ruber, P. punctatus) estuvieron mejor representados en horas diurnas y al atardecer. En algunos rangos horarios se capturaron muy pocos individuos de A. rhomboidalis, $H$. aurolineatum y $O$. ruber, por lo cual no fue posible incluirlos en el análisis de las estructuras de tallas (Fig. 6).
En la zona sur, F. notialis presentó un rango de tallas similar en los diferentes horarios examinados, pero tuvo una distribución de tallas más platicúrtica durante la noche (Fig. 7). La estructura de tallas de la comunidad de peces capturada en esta zona tuvo una mayor representación de tallas pequeñas $(4$ a 8 $\mathrm{cm})$ al amanecer que en los otros horarios $(\mathrm{p}<0,05)$ (Fig. 7). Aunque esta diferencia fue sutil, derivó en diferencias significativas en las distribuciones de 
Tabla 5. Nivel de significancia (p) en las pruebas de independencia $G$ de las distribuciones de tallas analizadas en la zona de pesca norte del Mar Caribe de Colombia entre julio y septiembre de 2004. 1, amanecer; 2, día; 3, atardecer; 4, noche. NA, la prueba no es aplicable. gl, grados de libertad.

Table 5. Significance level (p) in $\mathrm{G}$ independency tests of the analysed length distributions in the northern fishing zone of Colombian Caribbean Sea between July and September 2004. 1, at dawn; 2, daytime; 3, at dusk; 4, at night. NA, test is not aplicable. gl, degrees of freedom.

\begin{tabular}{l|cccccccc|cc}
\hline & \multicolumn{7}{|c}{ p } & \multicolumn{3}{|c}{ gl } \\
\cline { 2 - 10 } & Global & $\mathbf{1 v s 2}$ & $\mathbf{1 v s 3}$ & $\mathbf{1 - 4}$ & $\mathbf{2 - 3}$ & $\mathbf{2 - 4}$ & $\mathbf{3 - 4}$ & Global & Pares \\
\hline Captura total & 0,000 & 0,000 & 0,000 & 0,000 & 0,000 & 0,000 & 0,000 & 63 & 21 \\
Farfantepenaeus notialis & 0,000 & 0,000 & 0,000 & 0,000 & 0,000 & 0,000 & 0,000 & 27 & 9 \\
Farfantepenaeus brasiliensis & 0,000 & 0,000 & 0,000 & 0,000 & 0,000 & 0,000 & 0,000 & 15 & 5 \\
Descartes & 0,000 & 0,000 & 0,000 & 0,000 & 0,000 & 0,000 & 0,000 & 45 & 15 \\
Captura incidental & 0,000 & 0,000 & 0,000 & 0,000 & 0,000 & 0,000 & 0,459 & 42 & 14 \\
Lutjanus synagris & 0,000 & 0,000 & 0,049 & 0,001 & 0,005 & 0,018 & 0,085 & 10 & 5 \\
Diapterus spp. & 0,000 & 0,001 & 0,096 & 0,216 & 0,000 & 0,000 & 0,125 & 12 & 4 \\
Micropogonias furnieri & 0,000 & 0,000 & 0,001 & 0,000 & 0,001 & 0,001 & 0,057 & 21 & 7 \\
Orthopristis ruber & 0,000 & NA & NA & NA & 0,000 & 0,000 & 0,000 & 14 & 7 \\
Synodus spp. & 0,000 & 0,000 & 0,000 & 0,000 & 0,000 & 0,000 & 0,000 & 30 & 10 \\
Archosargus rhomboidalis & 0,000 & 0,000 & 0,294 & NA & 0,003 & NA & NA & 24 & 8 \\
Syacium spp. & 0,000 & 0,000 & 0,000 & 0,000 & 0,000 & 0,000 & 0,000 & 12 & 6 \\
Haemulon aurolineatum & 0,000 & NA & NA & NA & 0,000 & 0,000 & 0,000 & 15 & 5 \\
Prionotus punctatus & 0,000 & 0,000 & 0,000 & 0,007 & 0,000 & 0,000 & 0,000 & 15 & 5 \\
Upeneus parvus & 0,000 & 0,000 & 0,000 & 0,000 & 0,000 & 0,000 & 0,000 & 15 & 5 \\
Diplectrum spp. & 0,000 & 0,000 & 0,000 & 0,000 & 0,000 & 0,000 & 0,000 & 18 & 6 \\
Selene setapinnis & 0,000 & 0,000 & 0,000 & 0,000 & 0,000 & 0,000 & 0,000 & 27 & 9 \\
\hline
\end{tabular}

frecuencias de tallas entre los horarios (Tabla 6). La mayoría de la captura en la zona sur es desechada, por lo cual las distribuciones de tallas de los descartes mostraron un resultado similar. La captura incidental presentó individuos de tallas superiores a $10 \mathrm{~cm}$ y la fracción de tamaños menor (10 a $18 \mathrm{~cm})$ estuvo mejor representada en las capturas del amanecer (Fig. 7, Tabla 6).

La composición por tallas de los taxa dominantes en la zona sur evidenció diferencias leves a lo largo del día, con rangos de talla menores que los observados en la zona norte y concentrados en tamaños pequeños. Las frecuencias de tallas de L. synagris y Lepophidium spp. estuvieron distribuidas más homogéneamente al atardecer que en otros horarios (Fig. 8). No fue posible realizar el análisis de la estructura de talla de Dasyatis spp. (rayas) y Cynoscion acoupa (corvinata), porque el número de individuos capturado fue insuficiente para este propósito. 


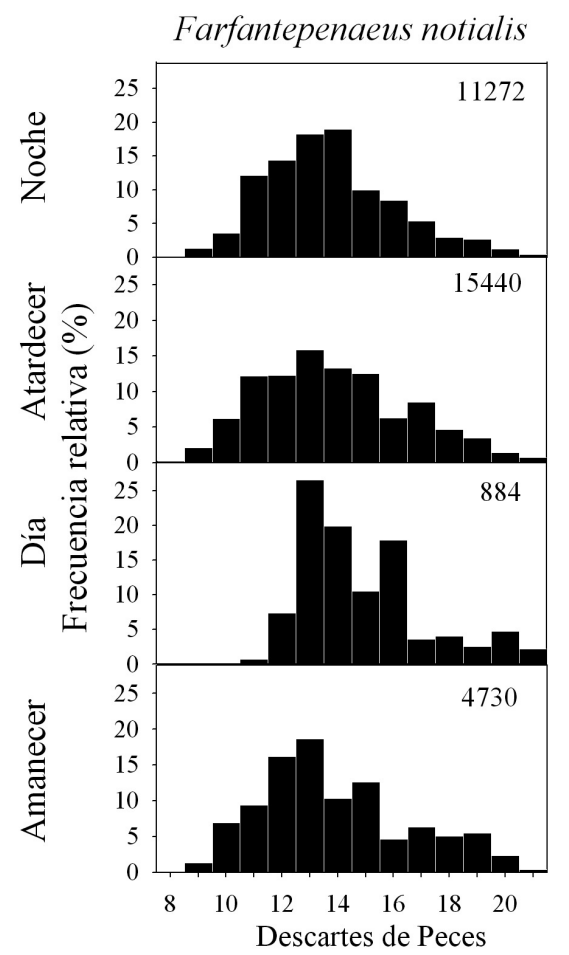

Farfantepenaeus brasiliensis
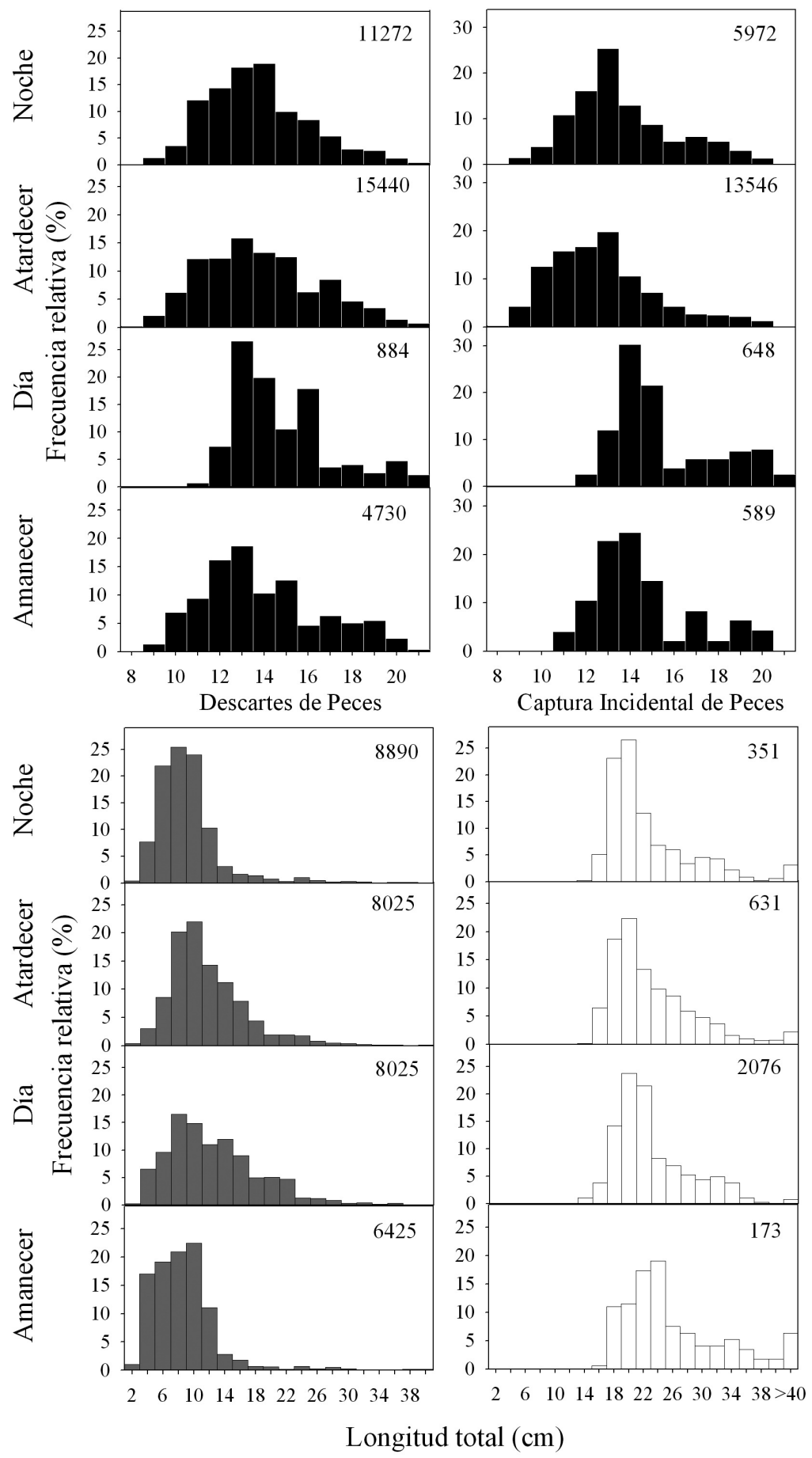

Figura 5. Variabilidad circadiana de las estructuras de tallas de la captura total de Farfantepenaeus notialis, $F$. brasiliensis, los descartes y la captura incidental observadas en la zona de pesca norte. Los datos se agruparon en rangos horarios que representan el amanecer, el día, el atardecer y la noche.

Figure 5. Circadian variability of size structures of total catch of Farfantepenaeus notialis, F. brasiliensis, discards and incidental catch observed in the northern fishing zone. Data were grouped in time ranges representing at dawn, daytime, at dusk and at night. 
Tabla 6. Nivel de significancia (p) en las pruebas de independencia $\mathbf{G}$ de las distribuciones de tallas analizadas en la zona de pesca sur del Mar Caribe de Colombia entre julio y septiembre de 2004. 1: amanecer, 3: atardecer, 4: noche. NA, la prueba no es aplicable. gl, grados de libertad.

Table 6. Significance level (p) in $G$ independency tests of the analysed length distributions in the southern fishing zone of Colombian Caribbean Sea between July and September 2004. 1: at dawn, 3: at dusk, 4: at night. NA, test is not aplicable. gl, degrees of freedom.

\begin{tabular}{l|cccc|cc}
\hline & \multicolumn{3}{|c}{$\mathbf{p}$} & \multicolumn{2}{c}{ gl } \\
\cline { 2 - 7 } & Global & $\mathbf{1 - 3}$ & $\mathbf{1 - 4}$ & $\mathbf{3 - 4}$ & Global & Pares \\
\hline Captura Total & 0,000 & 0,000 & 0,000 & 0,000 & 40 & 20 \\
Farfantepenaeus notialis & 0,000 & 0,000 & 0,000 & 0,000 & 22 & 11 \\
Descartes & 0,000 & 0,000 & 0,000 & 0,000 & 38 & 19 \\
Captura Incidental & 0,000 & 0,000 & 0,000 & 0,000 & 26 & 13 \\
Cathorops spixii & 0,000 & 0,000 & 0,000 & 0,000 & 22 & 11 \\
Diplectrum spp. & 0,000 & 0,000 & 0,000 & 0,000 & 10 & 5 \\
Eucinostomus argenteus & 0,000 & 0,005 & 0,000 & 0,000 & 18 & 9 \\
Lepophidium spp. & 0,000 & 0,000 & 0,000 & 0,000 & 16 & 8 \\
Lutjanus synagris & 0,000 & 0,004 & 0,003 & 0,000 & 16 & 8 \\
Porichthys plectrodon & 0,000 & 0,000 & 0,000 & 0,000 & 20 & 10 \\
Prionotus punctatus & 0,000 & 0,000 & 0,000 & 0,000 & 12 & 6 \\
Selene setapinnis & $\mathrm{NA}$ & $\mathrm{NA}$ & $\mathrm{NA}$ & $\mathrm{NA}$ & $\mathrm{NA}$ & $\mathrm{NA}$ \\
Syacium spp. & 0,000 & 0,000 & 0,000 & 0,000 & 10 & 5 \\
Synodus spp. & 0,000 & 0,000 & 0,000 & 0,000 & 28 & 14 \\
\hline
\end{tabular}



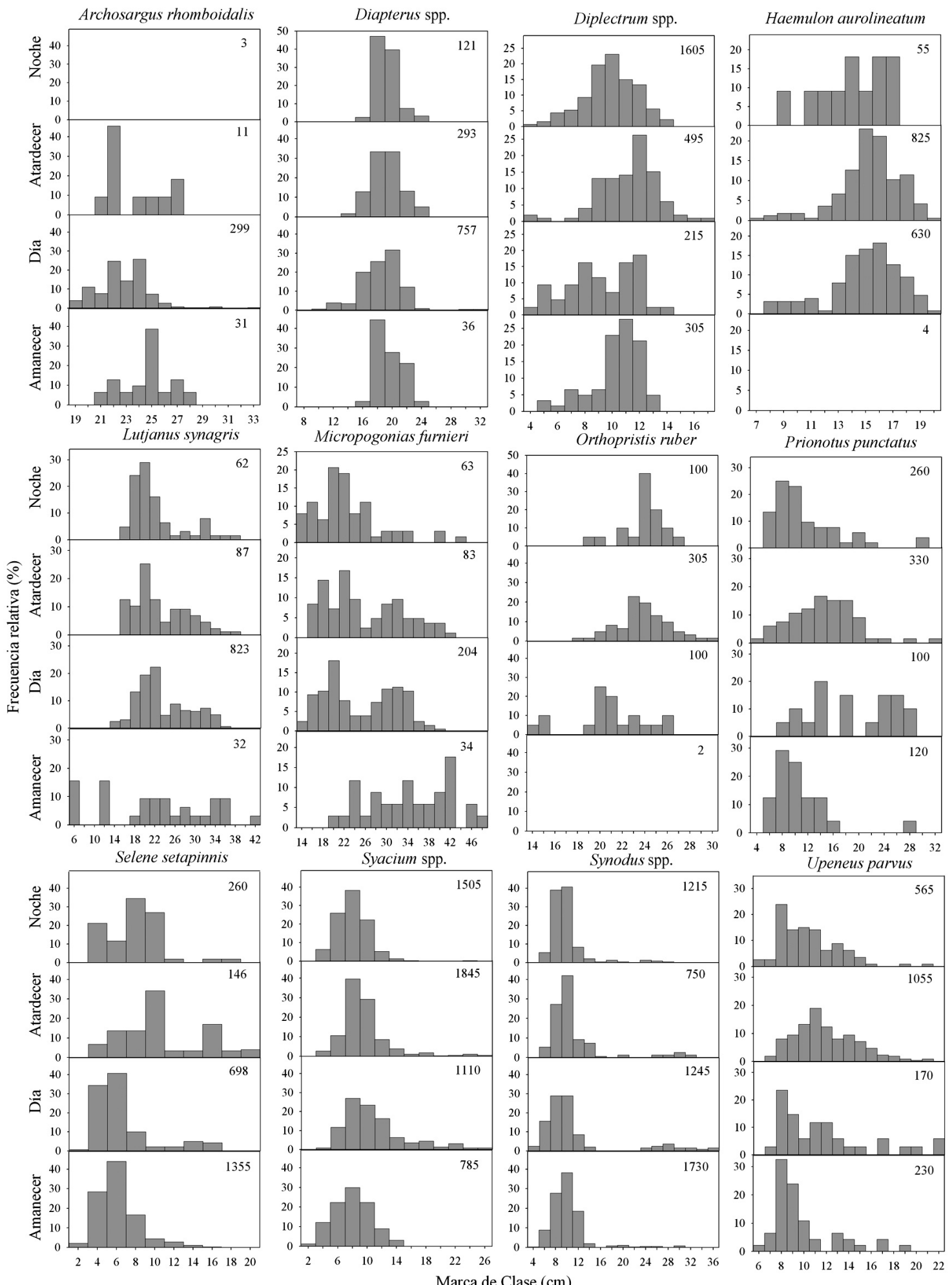

Figura 6. Variabilidad circadiana de las estructuras de tallas de los taxa de peces dominantes en la zona de pesca norte.

Figure 6. Circadian variability of size structures of dominant fish taxa in the northern fishing zone. 


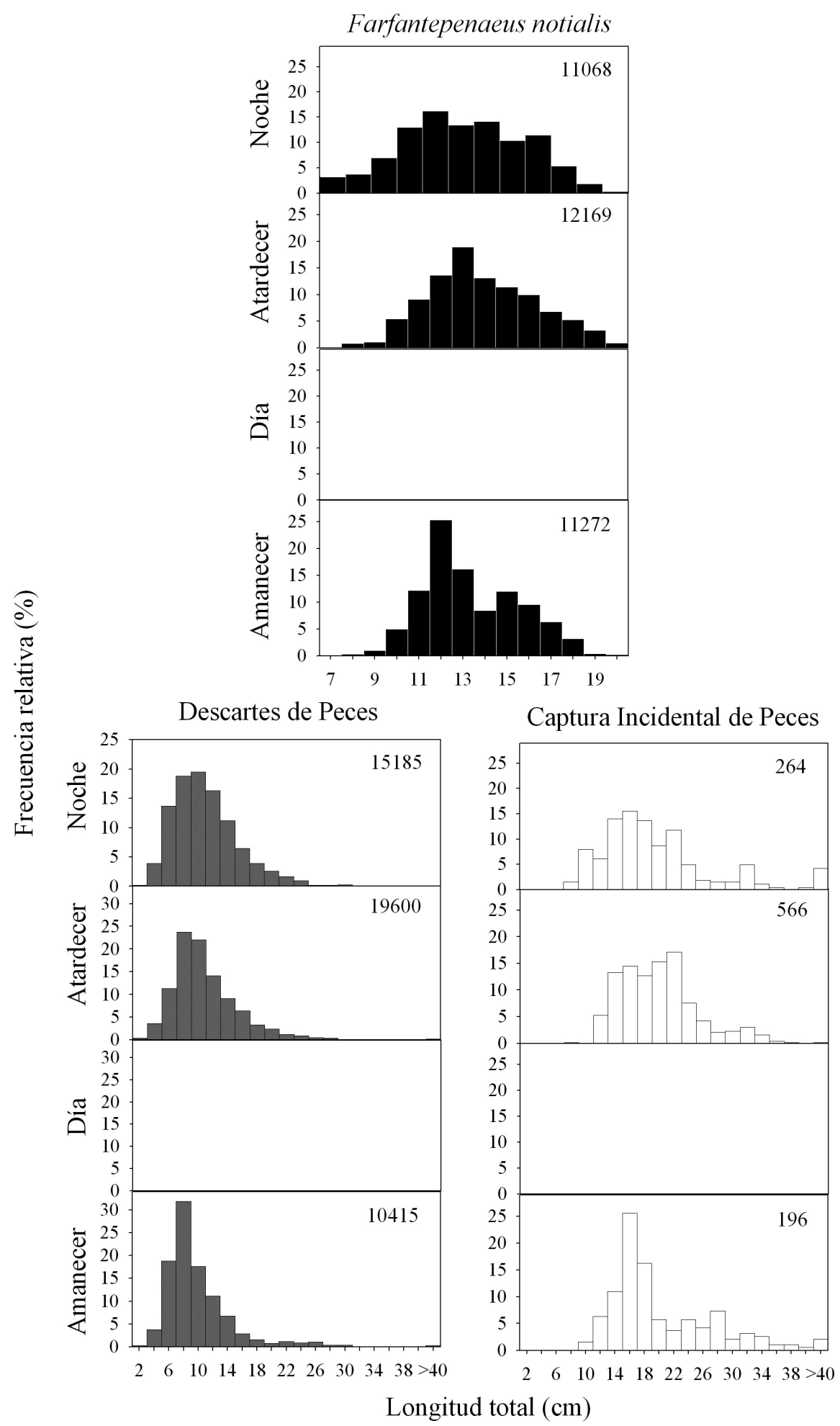

Figura 7. Variabilidad circadiana de las estructuras de tallas de la captura total de Farfantepenaeus notialis, los descartes y la captura incidental observadas en la zona sur.

Figure 7. Circadian variability of size structures of total catch of Farfantepenaeus notialis, discards and incidental catch observed in the southern fishing zone. 

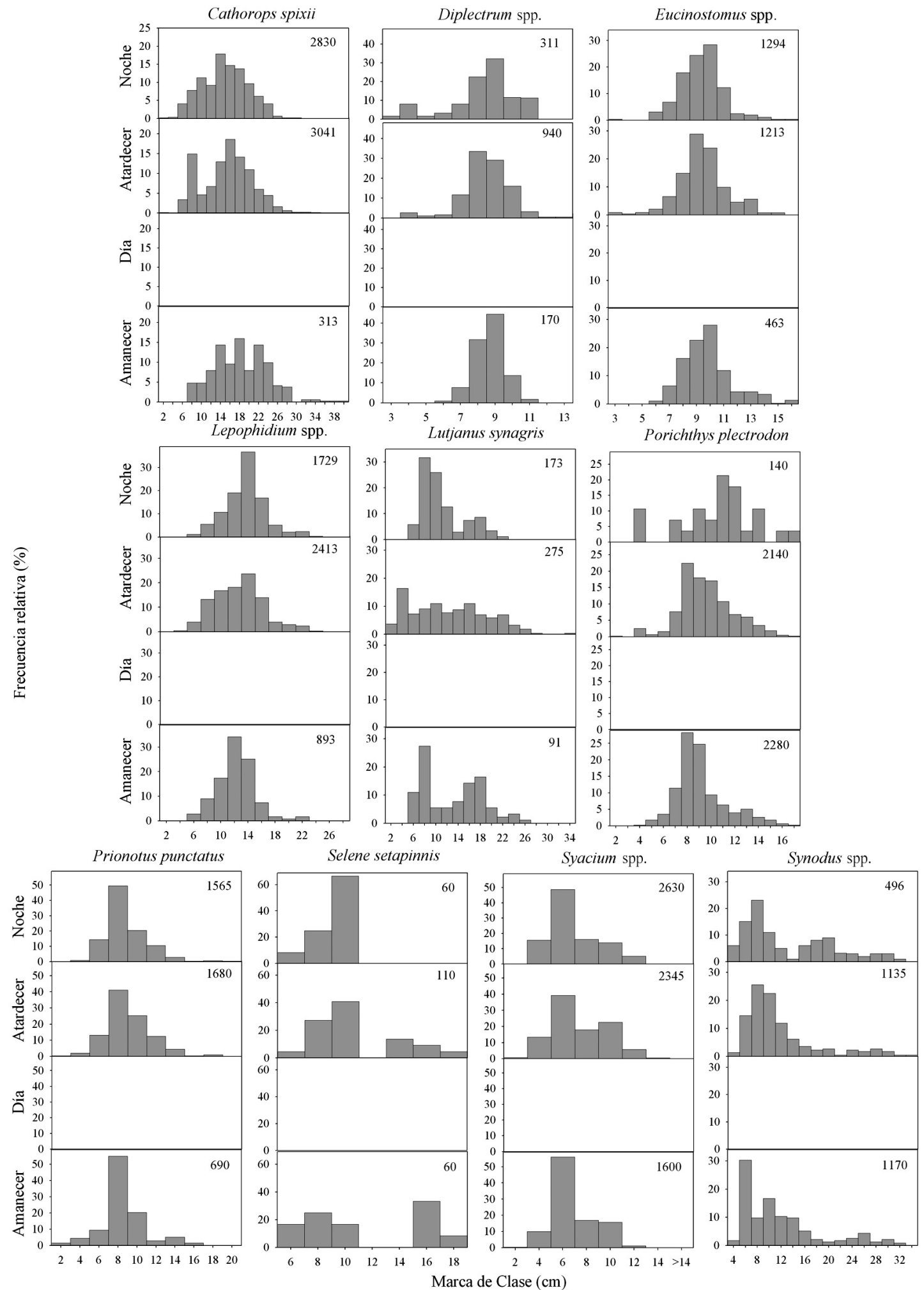

Figura 8. Variabilidad circadiana de las estructuras de tallas de los taxa de peces dominantes en la zona sur.

Figure 8. Circadian variability of size structures of dominant fish taxa in the southern fishing zone. 


\section{DISCUSIÓN}

Varios aspectos del comportamiento de los recursos pueden variar en el ciclo diurno y afectar las tasas de captura y la estructura de tamaño en las pesquerías de arrastre demersal. Se destacan las migraciones horizontales y verticales (Matthews et al., 1991; Pillar \& Barange, 1997; Aglen et al., 1999), el ocultamiento a depredadores (Steiner et al., 1982), detección visual y escape de la red de arrastre (Walsh \& Hickey, 1993) o comportamiento gregario (Walsh, 1991; Sierra et al., 1994). Los resultados obtenidos pueden ser principalmente explicados por la existencia de ritmos diurnos de actividad alimentaria de los depredadores y de escape de esa depredación por parte de las especies que son sus presas.

La variabilidad a lo largo del día de las tasas de captura en camarones ratifica la actividad nocturna que caracteriza la familia Penaeidae (e.g. Fuss \& Ogren, 1966), los cuales permanecen enterrados en el sedimento la mayor parte del día y sólo emergen en la noche para alimentarse, lo cual puede protegerles de los depredadores (Matthews et al., 1991) o de las altas temperaturas en horas diurnas (Hindely, 1975).

Las mayores tasas de captura de peces depredadores (L. synagris, M. furnieri, A. rhomboidalis) en horas diurnas en la zona norte (Tabla 1), se puede deber a su mayor disponibilidad a las redes de arrastre en el día, cuando forman agregaciones en las aguas someras en que opera la flota (a menos de $65 \mathrm{~m}$ de profundidad), mientras que en la noche se dispersan, incluso fuera de la zona de pesca, para alimentarse (Chavance et al., 1986; Sierra et al., 1994; Duarte \& García, 1999a). Aunque no se detectaron diferencias significativas en las tasas de captura de $H$. aurolineatum, hubo una tendencia a mayores valores en horario diurno (Tabla 1), que concuerda con las migraciones nocturnas a aguas profundas observadas en esta especie para alimentarse (Gaut \& Munro, 1983). La variabilidad circadiana en la actividad de los depredadores ha sido ampliamente documentada (Pillar \& Barange, 1997; Aglen et al., 1999; Yousif, 2003). Sin embargo, este resultado puede estar influido por el direccionamiento de algunos capitanes hacia la captura de fauna incidental en horario diurno para compensar los bajos rendimientos de camarón que recientemente se han observado. En la zona sur se observaron pocas diferencias en las tasas de capturas durante las horas examinadas, debido probablemente a que la flota opera sólo desde el atardecer hasta el amanecer, con lo cual no es posible observar cambios día-noche. No obstante, se destaca que la tasa de captura total (compuesta principalmente por especies descartadas) es significativamente mayor en horas de la noche que en las horas del amanecer y anochecer (Tabla 2). Esta variabilidad circadiana puede deberse a la mayor actividad nocturna que exhiben las especies demersales pequeñas que caracterizan la comunidad capturada en esa zona, como estrategia para escapar de los depredadores, tal como se ha sugerido en otras regiones (Benoit \& Swain, 2003).

La tendencia a una mayor proporción de ejemplares de tamaño pequeño de $F$. notialis y de la comunidad de peces se observó en la zona sur (Figs. 2 y 3 ). El predominio de tallas grandes en la zona norte explica la menor proporción de descartes que se observan en esa zona (Figs. 3 y 4), mientras que un $70 \%$ de las especies capturadas en la zona sur son descartadas. La flota opera a menores profundidades en la zona sur (3-25 m) que en la zona norte (25-65 $\mathrm{m})$, lo cual brinda una explicación al hecho que las capturas de $F$. notialis y las capturas comunitarias (i.e. el conjunto de las especies) presenten tallas más pequeñas pues esta fracción de tamaño comúnmente se asocia a fondos someros. En efecto, estudios previos de las comunidades demersales del Mar Caribe de Colombia han identificado a la profundidad como un factor determinante para la estructuración de la fauna (García et al. 1998; Manjarrés et al., 2001). El carácter estuarino de la zona sur brinda hábitat para juveniles de camarones, juveniles de diversas especies de peces marinos (e.g. Diapterus spp., $M$. furnieri, L. synagris) (Cervigón et al., 1992) y para especies pequeñas propias de este tipo de aguas (e.g. P. plectrodon) (Bowman et al., 2000). Se descarta la posibilidad de que la presencia de tallas mayores en la zona norte se deban a migraciones ontogenéticas proveniente de la zona sur ya que el río Magdalena actúa como una barrera geográfica, detectándose diferencias en los stocks de algunas poblaciones, incluso en distancias inferiores a la que separa las zonas de pesca (Gómez-Canchong et al., 2004) y los pescadores de la zona sur han observado una reducción drástica de las tallas en las capturas en tiempos recientes.

La ausencia de los tamaños más pequeños de las especies camarones (F. notialis y F. brasiliensis) en horas diurnas (Fig. 5), indica que la estrategia de ocultamiento que exhiben los camarones en horas diurnas (Fuss \& Ogren, 1966) es un mecanismo que favorece la protección de la fracción de tamaño más vulnerable a los depredadores, en contraposición del comportamiento de desenterramiento sincrónico de todos los miembros de la población en el ocaso que se 
ha propuesto (Hughes, 1969). El uso de la estructura del hábitat y el sustrato como refugio preferencial de juveniles de camarones ha sido documentado (Primavera, 1997).

La mayor proporción de individuos pequeños (particularmente descartes) en las capturas de la comunidad de peces en la zona norte al amanecer, atardecer y noche puede atribuirse a la actividad alimentaria que hace que todas las tallas estén disponibles al arrastre en horas de penumbra y en la noche (Francis \& Williams, 1995; Benoit \& Swain, 2003; Yousif, 2003), en tanto que los individuos de talla grande e intermedia que constituyen la captura incidental (mayormente depredadores) estuvieron mejor representados en horas diurnas (Fig. 5), lo cual concuerda con la explicación previa de variabilidad circadiana en la actividad alimentaria para los patrones observados en las tasas de captura.

$\mathrm{Si}$ bien las mayores tasas de captura en especies depredadoras (L. synagris, M. furnieri) se observaron en horas diurnas en la zona norte (Tabla 1), los ejemplares más grandes estuvieron menos representados en esos horarios (Fig. 6), lo cual sugiere que los ejemplares más grandes no hacen parte de las agregaciones de peces que se establecen en aguas someras en horarios diurnos, probablemente desplegando una actividad trófica más o menos constante durante el día por ser tallas menos vulnerables a depredadores diurnos (Sierra et al., 1994; Duarte \& García, 1999b). Este comportamiento dependiente del tamaño puede explicar la mayor proporción de individuos de mayor talla observados en horario diurno y de especies medianas (P. punctatus, Synodus spp.) y pequeñas ( $S$. setappinnis, Syacium spp.) al atardecer (Fig. 6).

El análisis de la variabilidad a lo largo del día de la estructura de tallas en la zona sur es menos concluyente, ya que la flota no operó en esta zona en horario diurno. Las diferencias en las distribuciones de frecuencia de tallas fueron sutiles entre los horarios examinados (Fig. 7). Como característica destacable en esta zona, la captura incidental presentó individuos significativamente menores que la zona norte (límite inferior: $10 \mathrm{~cm} v s .16 \mathrm{~cm}$ ), como consecuencia de que recientemente en la zona sur los pescadores empezaron a comercializar especies pequeñas (e.g. Eucinostomus spp.) ante la disminución de los rendimientos de camarones y peces de mayor tamaño. En los horarios muestreados, sólo las fracciones de tallas pequeñas de los depredadores ( $L$. synagris, Lepophidium spp.) fueron observadas en la zona sur, lo cual impide detectar los cambios diurnos en la actividad alimentaria relacionados con la talla que se observaron en la zona norte. Sin embargo, la distribución de tallas más homogénea de estas especies al atardecer sugiere un comportamiento que hace más disponible a la pesquería un rango más amplio de tallas durante el día.

Una serie de implicaciones para la evaluación de los recursos, el efecto selectivo de la pesca sobre los recursos y el manejo pesquero se derivan de este análisis de la variabilidad circadiana de las tasas de captura y estructura de tamaños. Considerando que las tasas de captura y la estructura de tamaño de varias especies analizadas varían significativamente respecto a la hora del día en los arrastres de fondo, los muestreos deben ser aleatorizados respecto esta variable para evitar sesgos en los índices de abundancia y se deben aplicar factores de corrección en la capturabilidad.

La tasa de captura promedio de los camarones en la zona norte durante la noche triplicó a la diurna, mientras que la tasa de captura promedio de fauna incidental en horario diurno duplicó a la nocturna. Adicionalmente, un amplio rango de tamaños de peces depredadores fue disponible a la pesquería en horario diurno. Por lo tanto, en términos de rentabilidad y de protección tanto del hábitat como de la fauna acompañante, es recomendable realizar sólo faenas nocturnas, en contraposición con la actividad de la pesquería que incluye faenas diurnas en la zona norte. La actividad intensiva diurna y nocturna de la pesquería de arrastre deteriora el ecosistema más rápidamente, y los rendimientos diurnos del recurso objetivo son significativamente inferiores.

Recientemente, los pescadores han empezado a comercializar individuos y especies de tallas mucho menores a las que explotaban hace unos años (e.g. Eucinostomus spp.). Esto revela una perturbación profunda en la composición de tamaños de la comunidad demersal. Cambios de este tipo han sido observados en otras regiones fuertemente explotadas por pesquerías de arrastre, que involucran la disminución de la abundancia de peces grandes de vida larga, cambios en la biodiversidad y el espectro de tamaños integrado de la comunidad (Pauly, 1988; Bianchi et al., 2000). La pesquería de arrastre está impactando un rango amplio de tallas de las poblaciones dominantes en el sistema, principalmente tallas pequeñas en la zona sur y tallas grandes en la zona norte, lo cual las hace vulnerables tanto a sobrepesca por reclutamiento como por crecimiento.

En el Mar Caribe de Colombia existen señales 
del deterioro de las comunidades marinas como la disminución de los volúmenes de camarón y peces desembarcados por diversas flotas en los últimos años (INPA, 2000) y el incremento en la duración de los lances, que en este estudio fue de $6 \mathrm{~h}$ en promedio en la zona norte aunque tradicionalmente eran de 4 h (Viaña \& Manjarrés, 2004). Esta situación es agudizada por la gran cantidad de individuos de tallas pequeñas que son descartados en las dos zonas de pesca, lo cual evidencia una elevada presión pesquera e indica la urgencia de establecer medidas de manejo para mitigar el impacto de la pesca de arrastre. Los resultados que aquí se presentan serán un aporte valioso para que el diseño de esas medidas (e.g. dispositivos reductores de fauna acompañante o áreas marinas protegidas) preserven las especies y tallas apropiadas en cada zona de pesca.

\section{AGRADECIMIENTOS}

Este estudio fue financiado por Instituto Colombiano para el Desarrollo de la Ciencia y la Tecnología COLCIENCIAS (Proyecto Código 1117-09-13273), Universidad del Magdalena, INCODER, CARTAPESCA S.A., PESBOCOL S.A. y Universidad Nacional. El Servicio Alemán de Intercambio Académico (Deutscher Akademisher Austausch Dienst, DAAD) financió parcialmente a Luis O. Duarte y Paul Gómez-Canchong mediante becas de doctorado.

\section{REFERENCIAS}

Aglen, A., A. Engås, I. Huse, K. Michalsen \& B.K. Stensholt. 1999. How vertical fish distribution may affect survey results. ICES J. Mar. Sci., 56: 345-360.

Alverson, D.L., M.H. Freeberg, J.G. Pope \& S.A. Murawski. 1994. A global assessment of fisheries bycatch and discards. FAO Fish. Tech. Pap., 339: $1-233$.

Andrade-Amaya, C.A. 2000. The circulation and variability of the Colombian Basin in the Caribbean Sea. Tesis Ph. D. University of Wales, Wales, 223 pp.

Benoit, H.P. \& D.P. Swain. 2003. Accounting for length and depth-dependent diel variation in catchability of fish and invertebrates in an annual bottom-trawl survey. ICES J. Mar Sci., 60: 1298-1317.
Bianchi, G., H. Gislason, K. Graham, L. Hill, X. Jin, K. Koranteng, S. Manickchand-Heileman, I. Paya, K. Sainsbury, F. Sanchez \& K. Zwanenburg. 2000. Impact of fishing on size composition and diversity of demersal fish communities. ICES J. Mar. Sci., 57: 558-571.

Bowman, R.E., C.E. Stillwell, W.L. Michaels \& M.D. Grosslein. 2000. Food of northwest Atlantic fishes and two common species of squid. NOAA Tech. Memo. NMFS-NE 155, 138 pp.

Broadhurst, M.K. 2000. Modifications to reduce bycatch in prawn trawls: A review and framework for development. Rev. Fish Biol. Fish., 10(1): 27-60.

Cervigón, F., R. Cipriani, W. Fischer, L. Garibaldi, M. Hendrickx, A.J. Lemus, R. Márquez, J.M. Poutiers, G. Robaina \& B. Rodríguez. 1992. Fichas FAO de identificación de especies para los fines de la pesca. Guía de campo de las especies comerciales marinas y de aguas salobres de la costa septentrional de Sur América. FAO, Rome, $513 \mathrm{pp}$.

Chavance, P., A. Yañez-Arancibia, D. FloresHernández, A.L. Lara-Domínguez \& F.A. Linares. 1986. Ecology, biology and population dynamics of Archosargus rhomboidalis (Pisces, Sparidae) in a tropical coastal lagoon system, southern Gulf of Mexico. An. Inst. Cienc. Mar. Limnol. UNAM, 13(2): 11-30.

Cook, R. 2003. The magnitude and impact of by-catch mortality by fishing gear. Ch 13 En: M. Sinclair \& G. Valdimarsson. Responsible Fisheries in the Marine Ecosystem. FAO, Rome, 448 pp.

Duarte, L.O. \& C.B. García. 1999a. Diet of the lane snapper, Lutjanus synagris (Lutjanidae), in the Gulf of Salamanca, Colombia. Carib. J. Sci., 36(1-2): 54-63.

Duarte, L.O. \& C.B. García. 1999b. Diet of the mutton snapper Lutjanus analis (Cuvier) from the Gulf of Salamanca, Colombia, Caribbean Sea. Bull. Mar. Sci., 55(2): 453-465.

Duarte, L.O., C.B. García, J. Altamar \& L. Manjarrés. 2004. Bottom trawl bycath of the shrimp fishery in the upwelling area off Colombia, Caribbean Sea. Current status and historical insights. En: L. Manjarrés (ed.). Dinámica espacio-temporal del ecosistema de afloramiento del área Bocas de Ceniza-Punta Espada (Caribe colombiano) y sus implicaciones para un régimen de pesca responsa- 
ble. Informe Técnico. UNIMAG, COLCIENCIAS, INCODER, INPA, UNAL. Santa Marta, 24 pp.

Dunn, O.J. 1958. Estimation of the means of dependent variables. Ann. Math. Stat., 29: 1095-1111.

Fogarty, M.J. \& S.A. Murawski. 1998. Large-scale disturbance and the structure of marine systems: fishery impacts on Georges Bank. Ecol. Appl., 8 (Suppl. 1): S6-S22.

Francis, M.P. \& M.W. Williams. 1995. Diel variation in trawl match rates of Pagrus auratus (Sparidae). Fish. Res., 24: 301-310.

Fuss, C.M. \& L.H. Ogren. 1966. Factors affecting activity and burrowing habits of the pink shrimp Penaeus duorarum Burkenroad. Biol. Bull., 130: 170-191.

García, C.B., L.O. Duarte \& D. von Schiller. 1998. Demersal fish assemblages of the Gulf of Salamanca, Colombia (Southern Caribbean Sea). Mar. Ecol. Prog. Ser., 174: 13-25.

Gaut, V.C. \& J.L. Munro. 1983. The biology, ecology and bionomics of the Grunts, Pomadasyidae. pp. 110-141. En: J.L. Munro (ed.). Caribbean coral reef fhery resources. ICLARM Studies and Reviews 7, International Center for Living Aquatic Resources Management, Manila, Philippines, 276 pp.

Gómez-Canchong, P., D.J. Rodríguez, J. Arévalo \& L. Manjarrés. 2004. Identificación de stocks de pargo rayado Lutjanus synagris (Linnaeus, 1758) en el área norte del Caribe Colombiano. pp. 155179. En: L. Manjarrés (ed.). Pesquerías demersales del área norte del Mar Caribe de Colombia y parámetros biológico-pesqueros y poblacionales del recurso pargo. INPA, COLCIENCIAS, Universidad del Magdalena. Santa Marta, 317 pp.

Greenstreet, S.P.R. \& S.J. Hall. 1996. Fishing and the ground-fish assemblage structure in the north-western North Sea: an analysis of long-term and spatial trends. J. Anim. Ecol., 65: 577-598.

Hall, S.J. 1999. The effects of fishing on marine ecosystems and communities. Black-Well Science. Oxford, 274 pp.

Hindely, J.P. 1975. Effect of endogenous and exogenous factors on the activity of the juvenile banana prawn Penaeus merguiensis. Mar. Biol., 29: 1-8.

Hughes, D.A. 1969. Factors controlling the time of emergence of pink shrimp, Penaeus duorarum. En:
M.N. Mistakidis,. (ed.) Proceedings of the World Scientific Conference on the biology and culture of shrimps and prawns, Mexico City. FAO Fish. Rep., 57(3): 589-1165.

Instituto de Pesca y Agricultura (INPA). 2000. Boletín estadístico pesquero colombiano 1999-2000. INPA, Instituto de Pesca y Acuicultura. Bogotá, $139 \mathrm{pp}$.

Kruskal, W.H. \& W.A. Wallis. 1952. Use of ranks in one-criterion variance analysis. J. Amer. Stat. Ass., 47: 583-621.

Manjarrés L., C.B. García \& A. Acero. 2001. Caracterización ecológica de las asociaciones de peces demersales del Caribe colombiano norte con énfasis en pargos (Lutjanidae). Bol. Inst. Inv. Mar., 30: 77-107.

Manjarrés, L., L.O. Duarte \& C.B. García. 2003. El ecosistema de afloramiento del Mar Caribe de Colombia: implicaciones para un régimen de pesca responsable. Cienc. Tecnol., Colombia, 21(3): 1423.

Matthews, T.R., W.W. Schroeder \& E. Stearns. 1991. Endogenous rhythm, light and salinity effects on postlarval brown shrimp, Penaeus aztecus Ivaes, recruitment to estuaries. J. Exp. Mar. Biol. Ecol., 154: 177-189.

Michalsen, K., O.R. Godø \& A. Fernö. 1996. Diel variation in the catchability of gadoids and its influence in the reliability of abundance. ICES J. Mar. Sci., 53: 389-395.

Neilson, J.D. \& R.I. Perry. 1990. Diel vertical migrations of marine fishes: an obligate or facultative process? Adv. Mar. Biol., 26: 115-168.

Pauly, D. 1983. Algunos métodos simples para la evaluación de los recursos pesqueros tropicales. FAO Doc. Téc. Pesca, 234: 1-49.

Pauly, D. 1988. Fisheries research and the demersal fisheries of Southeast Asia. En: J.A. Gulland (ed.). Fish population dynamics. John Wiley and Sons, Londres, pp. 329-348.

Pelletier, D. 1998. Intercalibration of research survey vessels in fisheries: a review and an application. Can. J. Fish. Aquat. Sci., 55: 2672-2690.

Pillar, S.C. \& M. Barange. 1997. Diel variability in bottom trawl catches and feeding activity of the Cape hakes off the west coast of South Africa. ICES J. Mar. Sci., 54: 485-499. 
Pitcher, T.J., R. Watson, R. Forrest, H. Valtysson \& S. Guennette. 2002. Estimating illegal and unreported catches from marine ecosystems: a basis for change. Fish Fish., 3: 317-339.

Primavera, H. 1997. Fish predation on mangrove-associated penaeids. The role of structures and substrate. J. Exp. Mar. Biol. Ecol., 215: 205-216.

Sazima, I. 1986. Similarities in feeding behaviour between some marine and freshwater fishes in two tropical communities. J. Fish Biol., 29: 53-65.

Sidak, Z. 1967. Rectangular confidence regions for the means of multivariate normal distributions. J. Amer. Stat. Ass., 62: 626-633.

Sierra, L.M., R. Claro \& O.A. Povova. 1994. Alimentación y relaciones tróficas. En: C. Claro (ed.) Ecología de los peces marinos de Cuba. Centro de Investigaciones de Quintana Roo, México, pp. 263-320.

Sokal R.R. \& F.J. Rohlf. 2000. Biometry. W.H. Freeman and Company, New York, 887 pp.

Steiner, W. W., J.J. Luczkovich \& B.L. Olla. 1982. Activity, shelter usage, growth and recruitment of juvenile red hake Urophycis chuss. Mar. Ecol. Progr. Ser., 7: 125-135.
Viaña, J. \& L. Manjarrés. 2004. Inventario y caracterización general de la flota industrial de arrastre que operó en la zona norte del Caribe colombiano durante el año 2000. En: L. Manjarrés (ed.). Pesquerías demersales del área norte del Mar Caribe de Colombia y parámetros biológico-pesqueros y poblacionales del recurso pargo. INPA, COLCIENCIAS, Universidad del Magdalena. Santa Marta, 317 pp.

Walsh, S.J. 1991. Diel variation in availability and vulnerability of fish to a survey trawl. J. Appl. Ichth., 7: 147-159.

Walsh, S.J. \& W.M. Hickey. 1993. Behavioural reactions of demersal fish to bottom trawls at various light conditions. ICES Mar. Sci. Symp., 196: 6876.

Ye, Y. 2002. Bias in estimating bycatch-to-shrimp ratios. Aquat. Living Res., 15: 149-154.

Yousif, A. 2003. Diel variability of size and catch rate of three fish species and three penaeid prawns in the NW Red Sea trawl fishery. Fish. Res., 63: 265-274. 